\title{
Convergence and divergence in biology and medicine $^{\mathrm{a}}$
}

\author{
Vadim Korablev $^{1 *}$, Sergei Kozyrev $^{2}$, and Pavel Yakutseni ${ }^{2}$ \\ ${ }^{1}$ Peter the Great St. Petersburg Polytechnic University, Institute of Physics, Nanotechnologies and \\ Telecommunications, 195251 Polytechnicheskaya st. 29, Russian Federation \\ ${ }^{2}$ Peter the Great St. Petersburg Polytechnic University, Advanced Research Center, 195251 \\ Polytechnicheskaya st. 29, Russian Federation
}

\begin{abstract}
A convergence of digital and physical worlds shapes capabilities of attaining a fundamentally new level of knowledge about molecular and system-level principles of functioning of both individual elements of living world matter, and the entire biosphere. Accordingly, there appear those growing points, which change the traditional types of specialists' employment beginning to get oriented to solving the key problems of ecological and food-supply security, problems of biologically renewable sources of energy and materials, biotechnologies and bionic applications in engineering and industry, and ultimately to the challenges of public health services and healthcare. The basic changes of labor market in the fields related to biology and medicine have been shown. The directions of application of computational methods for searching biologically inspired breakthroughs for engineering and industry have been given consideration. The presented data can be interesting for analyzing negative, neutral and positive scenarios of development of universities and other education research communities.
\end{abstract}

Beginning from mid-1990s a new situation has been formed - the basic social mandate consists in researches and developments associated with biosciences and medicine. It appears that after scientific revolution of early new time, industrial revolution of $18-19^{\text {th }}$ centuries, development of industrial society in the $20^{\text {th }}$ century, and resultantly, reaching the unbelievable standards of technical comfort, the major challenge of our epoch is a lengthy and healthy life.

\section{Development of biology and medicine as a major trend of our time}

An impressive specific feature of development of present-time biology is the shift of this science from a descriptive paradigm to the sphere of exact sciences - physics and mathematics. The result of development of new generations of instruments, methods of

${ }^{a}$ The work has been supported by project No.16.7002.2017/БЧ of the Ministry of Education and Science of the Russian Federation

* Corresponding author: korablev@spbstu.ru 
investigating the living world is a generation of knowledge changing the world of public health services and healthcare, bio-oriented engineering and industry. An important peculiarity of the process is as follows: long-term trend of increasing expenditures in the field of biosciences and medicine; extensive establishment of interdisciplinary associations of specialists in the field of physics, biology, medicine, chemistry, engineering, management and industry accompanied by an intensive involvement of digital technologies.

The data shown in Fig. 1 are quite indicative for characterizing the trends of expenditures for development of biosciences and medicine. Apparently, that for more than ten years already the federal budgeting in terms of a system of national institutes of public health services of the USA exceeds \$25 bln., budgeting of all fundamental and applied works on engineering hardly reaches $\$ 12$ bln., while the budgeting fluctuates in the limits of $\$ 6 \div 4$ bln. for the sectors of physics, environmental protection, computer science [1]. The amounts of governmental and private investments in the European Union in the same period were equal to: pharmaceuticals and biotechnologies, equipment and services for public health service - $\$ 114$ bln., process equipment - $\$ 95$ bln., automotive branch $-\$ 84$ bln., software and computer services - \$39 bln., electronic and electric equipment - \$38 bln., materials and chemicals - \$26 bln., aerospace and defense complex - \$24 bln. and further in descending order [2]. Thus, it is necessary to ascertain the fact that biology, bioinspired industry and medicine become the main customers and consumers of R\&D in XXI century instead of space and defense programs and electronics.

The other manifestation of dominating bio-oriented development is the bibliometric data from the countries of European Union and the USA. For instance, the analysis of Internet base SCImago Journal \& Country Rank (http://www.scimagojr.com) shows that the share of quoted documents, predominantly, the scientific articles in the field of medicine, biochemistry and molecular biology is nearly 38\%. This indicator equals $20 \%$ and decreases from $28 \%$ and $26 \%$ during 1996 and 2002, accordingly, for the domains of physics and engineering.

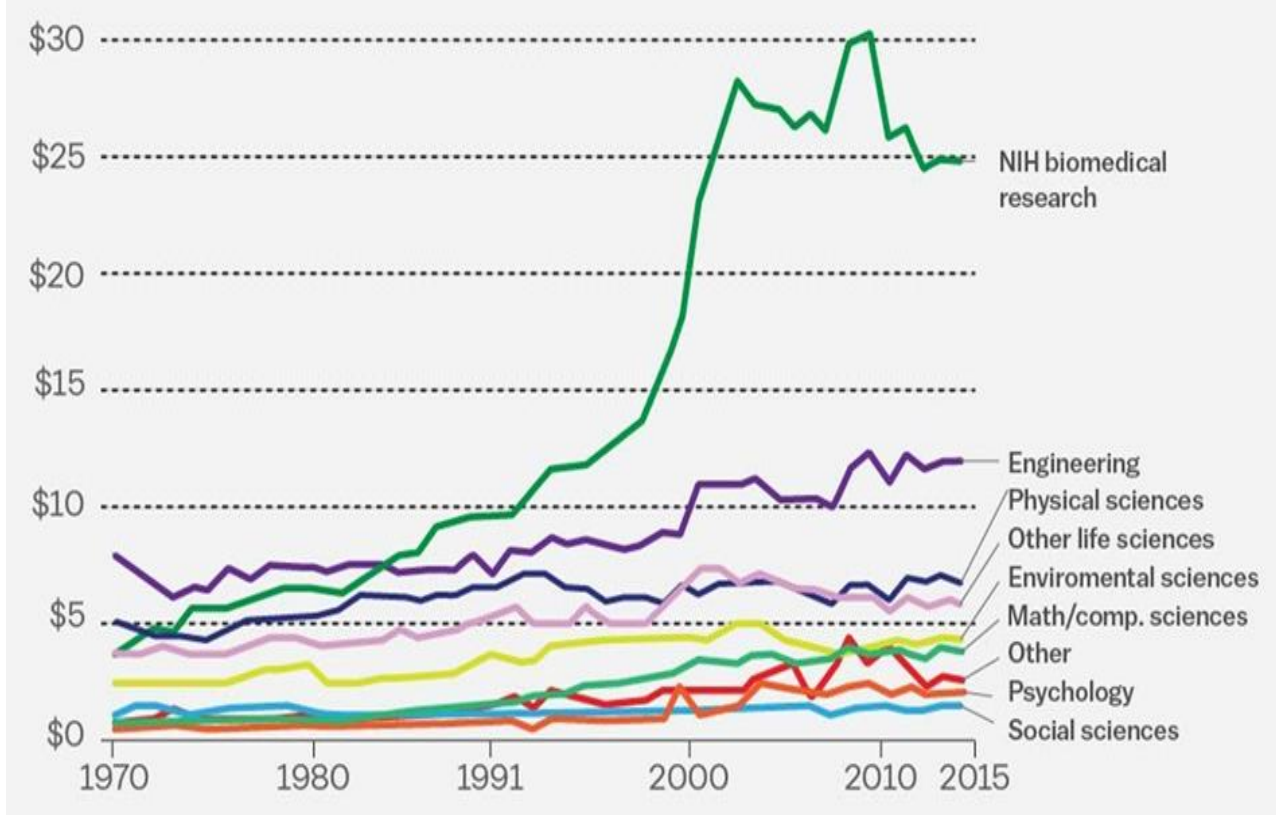

Fig. 1. Dynamics of federal expenses of the USA for scientific research and development by lines of activity. Data have been received from sources referred to in the work [2]. 
Without doubt, the emergence of the above trends is accompanied by structural changes in the leading Universities, even those, which positioned themselves as purely technical earlier. The Internet data in the majority of world higher educational institutions, including Russia, testify to a permanent drift to the field of medicine, biology and its applications. ARWU, THE and QS pertaining to biology and medicine occupy the first positions of divisions of the world ratings, Massachusetts Institute of Technology (MIT), California Institute of Technology, Zurich Institute of Technology and Federal School of Technology of Lausanne appear regularly on the charts. So, cumulative proceeds from the main activity of MIT in 2012 amounted to about \$3 bln., whereof the medical and biological works sponsored by the Government of the USA have already provided $23 \%$ of total financial turnover of MIT with $16 \%, 15 \%$ and $14 \%$ of orders associated with defense, industry and power engineering, accordingly. The share of sector of life science and medicine reaches $40 \div 50 \%$ for 2017 in the top-rated technical HEI of the advanced economies of the world. The department of natural sciences and mathematics of the Federal Institute of Technologies of Zurich consists of four divisions: biology, chemistry and applied biosciences, mathematics, physics. Technical University of Munich has its own hospital (34 clinical units for 1,100 beds) providing clinical investigations and translation of scientific discoveries into medical practice. Such practices are characteristic for the Universities of other countries of technological nucleus, China, region of Southeast Asia. During the last three years the leading Universities of Russia, first of all those that take part in the program of improving competitiveness "5-100-2020" set up bio-oriented research divisions and departments in their structure.

It is expected that the development of the line of activity will preserve the dominating character for decades in future and will be accompanied by intensive incorporation of those methods of computational data analysis, predictive simulation and virtual testing, which similarly to the systems of computer-aided design (CAD) will be able to provide the next interdisciplinary level of convergent creation of prototypes and technologies of bio-inspired prototypes and technologies of bio-inspired industry and medicine the same as half a century ago in engineering. Unfortunately, the present situation in the field of biology and medicine is extremely disengaged.

\section{Divergence in biology and medicine}

In comparison with the world of inanimate and technical systems, the hierarchical structure of biological objects is more than complex. Different levels are distinguished quite frequently in biology: atoms and molecules (1); simple bimolecular complexes (2); atom and molecular clusters and nanosystems (3); functional ensembles of atom and molecular clusters and nanostructures (4); subcellular formations and organelles (5); individual cell and cellular ensembles $(6,7)$; clusters of interacting cellular ensembles and tissues $(8,9)$; individual organs (10); systems of interrelated organs (11); organisms (12), groups thereof further to populations, biomes and right up to integral concepts of biosphere. At that, the poorly interacting cohorts of specialists get developed and resumed around every level. They get divided in turn both by the objects of research (proteins, nucleic acids, mitochondrions, lungs or other), and from the point of view of properties under investigation, like structure, biochemistry, functions and so on and so forth. After that differentiation of collectives and groups by applications begins: pharma industry, biotechnologies, medical equipment and so on.

The upside of such a situation is an unprecedented stream of emerging investigation technologies and data on split-level phenomena and processes of the world of biosystems, shaping revolutionary ideas, generating new sectors of markets of bioeconomics, employment and medical services. 
Unfortunately, today, when the flow of reports about biomedical breakthroughs has become almost daily, yesterday's seemingly most remarkable sensations turn out to be in the basket of past hopes. The enhancing crisis of hyperspecialization, increasing barriers of interdisciplinary communications and, hence, the accompanying destruction of systems of substantial financing and administration of science and education appear to be a negative side of the process of development of biosciences. There emerges a picture of associations, where the steady and directed development is replaced with the personal interests of the groups under conditions of multidimensional branch of human knowledge - bioscience and medicine. A sector encompassing the army of biologists, health professionals, physicists, engineers and computer experts falls to isolated detachments generating omnidirectional growth points, which under conditions of absence of comparative criterion are frequently deemed as consensuses of professionals. Here, at the frontier of breakthroughs we impersonally face a wide spectrum of ideas with limitedness of general resource of investigations and developments.

In general, facing a situation of almost the so-called "black box" we always see more than a multiplicity of development points: some are attracted by proteomics, the other are attracted by regenerative medicine and cell technologies, the third deal with metaanalysis or "BigData", and all together they are looking for those common factors, which will be able to predict general mechanisms of biosystems and a human being under normal conditions, in situations of illness and medical interventions. An important goal is the implementation of that approach, when "deliberation" of ideas is effected at the level of quantitative calculation, reaching that "growth superpoint", which existing in physics and engineering sciences makes it possible to combine knowledge of different specialists, substantiate prospects of projects, articles or structures.

\section{Digital and real experiment}

The models of objects and technologies are developed, expected properties are calculated and after that only the prototypes get created, which help assess the result in the course of real (full-scale) experiment in exact and engineering sciences in case of existing sufficient theory after appearance of the original idea.

In the life sciences we face a more complex reality. The depth processes of functioning biosystems still frequently remain in the field of vision of researchers, while the available models most frequently feature too much a primitive character. Therefore, generally, in biology and medicine one has to solve a problem of experiment interpretation, i.e. restoration of internal unobservable subject matter of events by their external manifestations. In the exact and engineering sciences such situation corresponds to a case of so called problems of diagnostics, "black box" approaches and other inverse problems. At that, the suchlike inverse problems in mathematics always belong to a class of ill-posed ones, and the solution thereof is either absent, or produces unstable or non-sole result. The cost of absence of predictive a priori models in biology is high today more than ever, since the volumes of works in the field of biology and medicine reach hundreds billions of dollars, the accumulated data arrays are counted in petabytes, the number of people involved in this sector grows by millions.

In scientific articles published in the field of exact sciences, typically, an opposite combination of approaches is used: theoretical simulation - real experiment. The simulation in modern physics and engineering provides for the following since long:

- $\quad$ exhaustive information capacity of data about multiple properties of systems under consideration, including those invariably unavailable for real observation;

- visualization of different-scale scenes of virtual reality; 
- consideration of external conditions unattainable in setting up a full-scale experiment and safety of investigations;

- $\quad$ predictive simulation providing for capabilities of directed designing and testing technologies and prototypes, substantiation of viability of the scheduled works;

- $\quad$ sharp reduction (reaching the orders of magnitude) of volumes, time limits and cost of works due to replacement of full-scale testing with digital experiments.

The methodology of bio-oriented investigations also begins to change. A triad customary to application-oriented physicists and engineers begins to be translated instead of traditional, extremely resource-intensive, and in view of the above peculiarities, solution of inverse problem and knowingly unreliable cascades of checks and re-checks: theoretical models; calculations according to these models; minimal test experiment. At that, the development of special methods of molecular and system-level simulation of split-level elements of a live world is required for biology and branches of medicine related thereto.

At the level of atoms and molecules we gradually transfer to considering ever more complex supermolecular structure, which makes it possible to obtain atomistically exact data about structure and interaction of both individual biologically important molecules, and complex ensembles of these substances, including molecules of water and ions of biometals. As a result we get not only ultimately detailed data on the position and motion of atoms and molecules in space and time, but also the data on energy jumps, thermodynamic, spectral properties and other easily measurable systems' properties, which makes it possible to supervise the authenticity of calculation. Practice shows that the accuracies of simulating small molecules appear to be higher than the errors of real experiment, while for macromolecules and nanobiostructures they are frequently comparable therewith [2].

Apparently, that the methods of system-level simulation acquire precedence in supermolecular hierarchically higher levels of organization of biosystems. For instance, the systems of differential levels connecting parameters characterizing the properties of systems/subsystems of the live world and variables reflecting current concentrations of different substances and intensity of processes of functioning of a separate organ or organism are given consideration. [2]

Acquisition of high-accuracy molecular pictures can determine the values of parameters of system-level models unknown in advance, which sharply increases the reliability of solutions. It should be noted that the present-day tools of molecular mechanics, semiempirical quantum mechanics, density functional and post Hartree-Fock metods make it possible to get exhaustive and reliable data, which can be used for building systemdefined higher models of recognition and biomechanics of cells, organs and hydrodynamic streams in biophase of living organisms, etc.

The cumulative application of methods of system-level and molecular simulation promise ever more split-level models of bioprocesses taking part on a spatial scale from atoms and molecules, cells and tissues to organisms and populations and time dynamics from picoseconds to days and years.

The successes of calculation approach have already brightly proven effective in investigating the level of nanomolecules and nanobiosystems. It is quite sufficient to mention here a field of molecular engineering and biocatalysts, materials and nanoprocesses de novo or designing medicines. Whereon the chemists have for years synthesized thousands of compounds, while the health professionals and biologists have tested these compounds for the role of new medicinal products, virtual models of the required biotargets appear, which make it possible to multiply reduce the volumes and time limits of experimental works, save up to $\$ 1 \mathrm{mln}$. per year for a project. Unfortunately, the fields of biology and medicine belong to a case, when direct simulation of the required multiscale objects (models of "digital" cell type, digital organ and digital man) is still inaccessible. Developing medicines, we fairly well forecast peculiarities of its absorption 
from the injection place, penetration to the place of action and molecular action on its target tissues, but further a complex reaction of different parts of tissues and organism arrives, most frequently predetermining a fuzzy, unexpected or unwanted result.

Presently, a direct simulation of biosystems using the methods of quantum or molecular mechanics is actually restricted by the levels of individual atom and molecular structures up to combinations thereof with the use of supercomputers. Further, a gap appears somewhere at the levels of cellular components, where are already unable to offer a substantial digital model and return to searching for empirical common factors of the "black box". There appears a necessity of solving two opposite problems. This, first of all, implies further development of methods of direct molecular simulation of more and more complex structures following a principle of emulation "bottom-upwards". Secondly, it implies a system-level (simulation) modeling of macroscopically observable systems and components thereof with the aim of combining the obtained results in the direction "top downwards".

\section{Convergence of competences}

The construction of individual proteins, deoxyribonucleic acid (DNA)/ ribonucleic acid (RNA), lipids, saccharides staying in isolated state or in liquid medium is being intensively investigated for many decades already. Consideration is given to biologically important micromolecules and complexes of micro- and macro-molecules, e.g., of ligand receptor or protein-protein type. The urge is there to distinguish one of the objects for simulation as one of the promising and undeveloped directions for development, viz., saccharides, frequently also referred to as carbohydrates, carbohydrates, polysaccharides or glycans. Glycans in line with proteins, lipids and nucleic acids correspond to one of four fundamental types of biomacromolecules and feature an individual spectrum of key functions associated with power-engineering, identification, structure formation and other processes of the living world [3]. They make about $66 \%$ of dry weight of the entire biosphere and accumulate about $70 \%$ of energy reserved thereby and are considered as a source alternative to oil for production of new materials and nanotechnologies of different purpose [4]. An unusual peculiarity of glycans is an outstanding structural versatility determining new orders in terms of density of information recording as compared with biopolymers of proteins, DNA/RNA. These substances make an important component of man's nutrition, but infrequently they interfere with the immune system functioning being a source of a number of other threats for health. It is supposed frequently with reference of an assemblage of the mentioned data that many functions of glycation remain yet undisclosed, while further development of glycobiology, in particular, simulation and investigation of molecular structures belonging to glycation can result in one of new scientific revolutions of our century, which is stated even in official documents prepared for the governments of the developed countries [4]. Simultaneously, during the last years, unlike genomics and proteomics, glycomics still remains to be the field of activity of comparatively narrow circle of professionals having published about 5 thousand articles against more than 200 thousand works dedicated to proteins, DNA/RNA, as it is indicated by our analysis of bibliographical base of PubMed for 2017. Supposedly, we defend the next position of convergent development of molecular biosciences. It is really so, It is not very easy for any physics university in this niche, but is comparatively simpler to attain a considerable breakthrough. Though, in the event it is just an example. It is important that glycans are always present in DNA/RNA, in more than a half of proteins and frequently in lipids. A differentiation of biomolecules by types, the same as any differentiations, appears to be quite conventional, while a combination of different implications gives frequently an unexpected result. 
In terms of convergence, apparently, more reasonable is the consolidated investigation of biomolecular systems not uniform in structure, integral nanobiostructures. The experimental data on the structure and functional properties of such systems are not numerous, indirect and inaccurate. The emulation procedures of atomistically exact simulation describing detailed state of such complex functional systems as "bottomupwards" quickly encounters the limited power of the used computers. The simulation models making it possible to describe behavior without simulating "top downwards" conditions provide for the use of multiplicities of knowingly undefined, poorly controlled parameters.

A similar situation can be observed also at the other levels of hierarchy of knowledge (Ref. items 1-12, first paragraphs, section 2). In general in the system-level biology and medicine we frequently make out the systems of complex differential equations, but we face not only big quantities of variables, like concentration of substances, time, etc., and a great number of unknown parameters such as constant of rate of substances transfer or reactions, etc.

It appears that the exit from the existing situation is quite evident and this is rather a preparation of goal-oriented projects and convergent specialists capable of consolidating experimental knowledge received in vitro, in vivo, plan and carry out digital experiments in silico based on the opposite methods of emulations/simulations than divergent development of biosciences following a principle of seed financings. In other words, to work in the framework of multiscale modellinge, where, for instance, it is possible at atomistical level of molecular subsystems to calculate primary constants of speed or affinity, build models of complex supermolecular systems and even more hierarchically high systems/super systems of different types, which promises basic changes of our perceptions of fundamental mechanisms of functioning living world, development of diseases, methods of searching for means of diagnostics and therapy, directions of developing nanobionics, biotechnologies and bioengineering of the nearest and long-term future.

\section{Scenarios of changing employment of scientific society}

According to official sources of the USA in the field of life science (science and engineering only) 286, 400 specialists have been employed as of 2016 [5], while the total number of people employed in the leading biomedical clusters of the country only exceeded one mln. people. According to the data of British statistics 86,900 researchers and development experts have been employed as of 2014 in London alone [6], in this case it is considered that every specialist of the sector is provided with an advanced financing and provides today the employment of about two and half specialists of allied professions (clinical medicine, physics, engineering, computer technologies, mathematics, etc.). In dynamics it means that if during a decade the number of sector's specialists increases by $34 \%$ or $64 \%$ (big or medium and small companies of London accordingly), then in the other sectors of science and engineering rather significant re-orientation of scientific and engineering services will be observed in the rest sectors of science and engineering, which denotes a global shift of $25-30 \%$ in the sector of employment of 5-6 mln. of scientists of the world.

Probably that the main social mandate, raised before scientists and development experts in the field of biomedical investigations is associated to a great extent with the increase of lifespan. The emergence of ageing contingents of population denotes a growing number of patients suffering of two-three chronic diseases simultaneously, at a time when pharmacotherapy of a single disease gives a positive result in less than $60 \%$ of cases even for a non-aged diseased person [7], which implies a practical impossibility to help. At that, in Russia [8] the same as in the other countries of the world due to a problem the load on 
clinics increases permanently, which only aggravates the social and economical situation of the majority of countries.

It appears that this situation will exercise further pressure on the groups, which continue to use methods of intuitive trials and errors traditional for the sector, unfortunately, still capable of providing maximum possible durability, number and financing of employees engaged in fulfillment of any project. It is expected that the same as in computational physics and engineering of the past ages, the snowballing process of forming digital biology and medicine will develop, when every success attracts tens and then thousands of allied scientists and development experts, which will require solution of the whole number of scientific and educational problems related to the emergence of required for still divergent today biosciences and developments of convergent specialists accumulating capabilities of convergence and translation of technology of different applied and fundamental sciences.

\section{References}

1. Survey of Federal Funds for Research and Development (National Center for Science and Engineering Statistics, Alexandria, 2017)

2. A. Rudskoi, S. Kozyrev, P. Yakutseni, Proceedings of the II-nd international scientific conference "Technological Prospects in the Framework of the Eurasian Space: New Markets and Economical Growth Points" (2016)

3. A. Varki et al., Essentials of glycobiology (Cold Spring Harbor Laboratory Press, New York, 2017)

4. Transforming Glycoscience: A Roadmap for the Future. (Committee on Assessing the Importance and Impact of Glycomics and Glycosciences, Board on Chemical Sciences and Technology, Board on Life Sciences, Division on Earth and Life Studies, National Research Council, 2012)

5. J. F. Sargent, CRS Report R43061 (Congressional Research Service, Washington, D.C., 2017)

6. Numbers of workplaces and of employees in Life Sciences and Healthcare in London, 2001-2014 [online], Available at:

(https://www.ons.gov.uk/employmentandlabourmarket/peopleinwork/employmentande mployeetypes/adhocs/numbersofworkplacesandofemployeesinlifesciencesandhealthcar einlondon20012014 (2015)

7. N. J. Schork, Nature, 350 (2015)

8. M. B. Denisenko, E. Ya. Varshavskaya, Economical Journal of the Higher School of Economics, 21 (2017) 